\title{
Chapel Hill bisphenol A expert panel consensus statement: Integration of mechanisms, effects in animals and potential to impact human health at current levels of exposure
}

\author{
Frederick S. vom Saal ${ }^{\star}$, \\ Division of Biological Sciences, University of Missouri-Columbia, 105 Lefevre Hall, Columbia, MO \\ 65211, United States
}

\section{Benson T. Akingbemi,}

Department of Anatomy, Physiology and Pharmacology, Auburn University, Auburn, AL 36849, United States

\section{Scott M. Belcher,}

Department of Pharmacology and Cell Biophysics, Center for Environmental Genetics, University of Cincinnati, Cincinnati, OH 45267, United States

\section{Linda S. Birnbaum,}

U.S. Environmental Protection Agency, Research Triangle Park, NC 27709, United States

\section{Andrew Crain,}

Biology Department, Maryville College, Maryville, TN 37804, United States

\section{Marcus Eriksen,}

Algalita Marine Research Foundation, Los Angeles, CA 90034, United States

Francesca Farabollini,

Department of Physiology, University of Siena, 53100 Siena, Italy

\section{Louis J. Guillette Jr,}

Department of Zoology, University of Florida, Gainesville, FL 32611, United States

\section{Russ Hauser,}

Department of Environmental Health, Harvard School of Public Health, Boston, MA 02115, United States

\section{Jerrold J. Heindel,}

Division of Extramural Research and Training, National Institute of Environmental Health Sciences, Research Triangle Park, NC 27709, United States

Shuk-Mei Ho,

Department of Environmental Health, University of Cincinnati Medical School, Cincinnati, OH 45267, United States

\section{Patricia A. Hunt,}

School of Molecular Biosciences, Washington State University, Pullman, WA 99164, United States Taisen Iguchi,

National Institutes of Natural Science, Okazaki Institute For Integrative Bioscience Bioenvironmental Science, Okazaki, Aichi 444-8787, Japan

\footnotetext{
(C) 2007 Published by Elsevier Inc.

*Corresponding author. Tel.: +1 573882 4367; fax: +1 573884 5020. vomsaalf@ missouri.edu (F.S. vom Saal).
} 


\section{Susan Jobling, \\ Department of Biological Sciences, Brunel University, Uxbridge, Middlesex, UK}

Jun Kanno,

Division of Cellular \& Molecular Toxicology, National Institute of Health Sciences, Tokyo 158-8501, Japan

Ruth A. Keri,

Department of Pharmacology, Case Western Reserve University School of Medicine, Cleveland, $\mathrm{OH}$ 44106, United States

Karen E. Knudsen,

Department of Cell and Cancer Biology, University of Cincinnati College of Medicine, Cincinnati, $\mathrm{OH}$ 45267, United States

Hans Laufer,

Department of Molecular and Cell Biology, University of Connecticut, Storrs, CT 06269, United States

Gerald A. LeBlanc,

Department of Environmental and Molecular Toxicology, North Carolina State University, Raleigh, NC 27695, United States

\section{Michele Marcus,}

Department of Epidemiology, Rollins School of Public Health, Emory University, Atlanta, GA 30322, United States

\section{John A. McLachlan,}

Center for Bioenvironmental Research, Tulane and Xavier Universities, New Orleans, LA 70112, United States

John Peterson Myers,

Environmental Health Sciences, Charlottesville, VA 22902, United States

Angel Nadal, Instituto de Bioingeniería, Universidad Miguel Hernández, Elche 03202, Alicante, Spain

Retha R. Newbold,

Laboratory of Molecular Toxicology, National Institute of Environmental Health Sciences, Research Triangle Park, NC 27709, United States

Nicolas Olea, CIBERESP Hospital Clinico-University of Granada, 18071 Granada, Spain

Gail S. Prins,

Department of Urology, University of Illinois at Chicago, Chicago, IL 60612, United States

Catherine A. Richter, USGS, Columbia Environmental Research Center, Columbia, MO 65201, United States

Beverly S. Rubin, Department of Anatomy and Cellular Biology, Tufts Medical School, Boston, MA 02111, United States

\section{Carlos Sonnenschein,}

Department of Anatomy and Cellular Biology, Tufts University School of Medicine, Boston, MA 02111, United States

Ana M. Soto,

Department of Anatomy and Cell Biology, Tufts University School of Medicine, Boston, MA 02111, United States 
Chris E. Talsness,

Charité University Medical School Berlin, Campus Benjamin Franklin, Institute of Clinical

Pharmacology and Toxicology, Department of Toxicology, 14195 Berlin, Germany

John G. Vandenbergh,

Department of Zoology, North Carolina State University, Raleigh, NC 27695, United States

Laura N. Vandenberg,

Tufts University Sackler School of Graduate Biomedical Sciences, Boston, MA 02111, United States

Debby R. Walser-Kuntz, Carleton College, Department of Biology, Northfield, MN 55057, United States

Cheryl S. Watson,

Biochemistry and Molecular Biology Department, University of Texas Medical Branch, Galveston, TX 77555, United States

Wade V. Welshons,

Department of Biomedical Sciences, University of Missouri, Columbia, MO 65211, United States

Yelena Wetherill, and

Department of Epidemiology, Harvard School of Public Health, Boston, MA 02115, United States

R. Thomas Zoeller

Biology Department, University of Massachusetts, Amherst, MA 01003, United States

\section{Keywords}

Bisphenol A; In vitro; In vivo; Rat; Mouse; Aquatic animal; Cancer; Low dose; Non-monotonic doseresponse curves; Developmental programming

\section{Introduction}

This document is a summary statement of the outcome from the meeting: "Bisphenol A: An Examination of the Relevance of Ecological, In vitro and Laboratory Animal Studies for Assessing Risks to Human Health" sponsored by both the NIEHS and NIDCR at NIH/DHHS, as well as the US-EPA and Commonweal on the estrogenic environmental chemical bisphenol A (BPA, 2,2-bis(4-hydroxyphenyl)propane; CAS\# 80-05-7). The meeting was held in Chapel Hill, NC, 28-30 November 2006 due to concerns about the potential for a relationship between BPA and negative trends in human health that have occurred in recent decades. Examples include increases in abnormal penile/urethra development in males, early sexual maturation in females, an increase in neurobehavioral problems such as attention deficit hyperactivity disorder (ADHD) and autism, an increase in childhood and adult obesity and type 2 diabetes, a regional decrease in sperm count, and an increase in hormonally mediated cancers, such as prostate and breast cancers. Concern has been elevated by published studies reporting a relationship between treatment with "low doses" of BPA and many of theses negative health outcomes in experimental studies in laboratory animals as well as in vitro studies identifying plausible molecular mechanisms that could mediate such effects. Importantly, much evidence suggests that these adverse effects are occurring in animals within the range of exposure to BPA of the typical human living in a developed country, where virtually everyone has measurable blood, tissue and urine levels of BPA that exceed the levels produced by doses used in the "low dose" animal experiments.

Issues relating to BPA were extensively discussed by five panels of experts prior to and during the meeting, and are summarized in five reports included in this issue: (1) human exposure to 
bisphenol A (BPA) [1]; (2) in vitro molecular mechanisms of bisphenol A action [2]; (3) in vivo effects of bisphenol A in laboratory animals [3]; (4) an ecological assessment of bisphenol A: evidence from comparative biology [4]; (5) an evaluation of evidence for the carcinogenic activity of bisphenol A [5]. Further discussion occurred at the meeting where participants from the panels were reorganized into four breakout groups. The consensus statements from the meeting are presented below.

The definition of "low dose" of BPA at this meeting used the same two criteria established at a prior NIH meeting concerning the low dose endocrine disruptor issue [6]: (1) for laboratory animal studies "low doses" involved administration of doses below those used in traditional toxicological studies conducted for risk assessment purposes. For BPA the lowest dose previously examined for risk assessment purposes was $50 \mathrm{mg}\left(\mathrm{kg}^{-1} \mathrm{day}^{-1}\right)$ in studies with rats and mice. The $50 \mathrm{mg}\left(\mathrm{kg}^{-1} \mathrm{day}^{-1}\right)$ dose is the currently accepted lowest adverse effect level (LOAEL) that was used to calculate the current US-EPA reference dose (the daily dose that EPA calculates is safe for humans over the life-time) of $50 \mu \mathrm{g}\left(\mathrm{kg}^{-1} \mathrm{day}^{-1}\right)$. The current reference dose is thus based on "high dose" experiments conducted in the 1980s [7]. (2) "Low dose" also refers to doses within the range of typical human exposure (excluding occupational exposures). For purposes of this meeting, the published literature that was reviewed met both of these criteria for being considered within the "low dose" range.

Hundreds of in vitro and in vivo studies regarding the mechanisms and effects of low doses of BPA, as well as studies of biomonitoring and sources of exposure, have been published in peer reviewed journals over the last 10 years, since the first "low dose" BPA in vivo studies were published [8-10]. The meeting was convened specifically to integrate this relatively new information. This task required the combined expertise of scientists from many different disciplines, and care was taken to ensure that participants covered these diverse areas.

BPA is a high-volume ( $>6$ billion pounds per year) production chemical used to make resins and polycarbonate plastic [11]. Of particular concern is the use of BPA in food and beverage plastic storage and heating containers and to line metal cans. In addition, potential environmental sources of BPA contamination are due to use in dental fillings and sealants [12], losses at the production site [13], leaching from landfill [14,15], and presence in indoors air [16].

BPA has become a chemical of "high concern" only in recent years, even though BPA was shown to stimulate the reproductive system in female rats and thus to be an "environmental estrogen" in 1936 [17], long before it was used as the monomer to synthesize polycarbonate plastic and resins in the early 1950s. However, more recent evidence has shown that BPA also exhibits other modes of endocrine disruption in addition to binding to estrogen receptors, such as alterations in endogenous hormone synthesis, hormone metabolism and hormone concentrations in blood. BPA also results in changes in tissue enzymes and hormone receptors, and interacts with other hormone-response systems, such as the androgen and thyroid hormone receptor signaling systems. While BPA was initially considered to be a "weak" estrogen based on a lower affinity for estrogen receptor alpha relative to estradiol [18], research shows that BPA is equipotent with estradiol in its ability to activate responses via recently discovered estrogen receptors associated with the cell membrane [19-22]. It is through these receptors that BPA stimulates rapid physiological responses at low picogram per ml (parts per trillion) concentrations. 


\section{Purpose and organization of the BPA meeting}

\subsection{Topic-focused expert panels}

To address the strength of the evidence regarding the published BPA research, an organizing committee was formed, and five panels of experts from different disciplines were established. Each panel had a chair or co-chairs and included a scientist who agreed to be primarily responsible, along with the chair, for preparing a preliminary draft of the panel's report. A web site was established on which all of the available electronic files of articles concerning BPA were posted, along with other pertinent information relating to the meeting. Prior to the meeting, the panel members began working on draft reports and communicated via electronic media and telephone conference calls. The resulting preliminary report from each panel was posted on the web site and distributed at the meeting for all participants to read. After the meeting, each panel completed a manuscript that is a part of this meeting report. These five panel reports were peer reviewed using the normal manuscript submission process to Reproductive Toxicology. The following specific concerns about BPA led to the five expert panels being established:

1. Leaching of BPA occurs from the resin lining of metal cans and from plastic food and beverage containers under conditions of normal use. BPA is also detected in water and air samples.

2. Parts per billion (ppb) levels of BPA that are unconjugated (not metabolized and thus biologically active) are detected in human blood and tissues in different countries, and these levels appear to be higher than blood levels that would be present in animals exposed to the US-EPA reference dose.

3. BPA causes a wide range of adverse effects at "low doses" that are below the USEPA reference dose in animals, both terrestrial and aquatic.

4. There is evidence from in vitro mechanistic studies that indicates the potential for disruption of human and animal cell function at concentrations of BPA far below unconjugated levels typically found in human blood and tissues.

5. There is evidence that at very low doses, BPA may be carcinogenic or increase susceptibility to cancer in animals.

The five panels each addressed a different topic related to their specific area of expertise with BPA and prepared a panel report that included documentation of the relevant published studies:

Panel\$durces and amounts of human exposure to BPA as well as pharmacokinetics.

Panel/(22)itro studies related to the molecular mechanisms that mediate responses to BPA with an emphasis on studies using low doses.

Panel[(B) ivo studies of BPA at "low doses" in laboratory animals.

Panel((4)yivo studies of BPA in aquatic wildlife and laboratory animals.

PanelR(5)ationship of BPA to cancers.

The purpose of the 3-day meeting was to provide an opportunity for members of the different panels to interact with each other to integrate information from different disciplines concerning low dose effects of BPA after each panel of experts had prepared a report in its specific area. The agenda of the meeting was designed to allow the members of the five panels to have time to discuss the information in their panel reports and finalize statements about the strength of the evidence for the literature that the panel had reviewed. 


\subsection{Integration of information by breakout groups}

For the second part of the meeting the focus was on integrating the information from each of the panel reports. This was accomplished by assigning panel members to one of four breakout groups. The four replicate breakout groups were established using the following criteria, such that each breakout group should have

1. At least two members from each of the five panels.

2. A person from each panel who had published on BPA.

3. A person with general knowledge of endocrine disruption research or endocrinology, but who had not necessarily published on BPA.

4. A person with experience in the process of reaching consensus.

5. A mixture of junior and senior investigators.

The charge to the replicate breakout groups was to individually integrate the information relating to the following four issues:

Issue Dattermine the degree to which the findings on BPA mechanisms of action identify mechanisms and bioactive doses that explain results of the studies reported by the panel on in vivo laboratory animal studies. Determine the strength of the evidence for plausible mechanisms mediating in vivo effects at low doses. In addition, identify any in vivo findings that are unexpected based on the in vitro literature.

Issue (2)sess the degree to which ecological studies with wildlife are consistent with laboratory studies in similar and different species. For example, determine the similarity of exposure levels and types of responses seen in wildlife and laboratory animals.

Issue (Bi)scuss the degree to which the low doses of BPA used in laboratory animal studies relate to the levels detected in human serum and tissues (including urine).

Issue sess the importance of life stage in the pharmacokinetics of BPA, levels of exposure to BPA, and the health effects of BPA in animals and humans.

\section{Findings submitted by the four breakout groups}

The reports from the breakout groups are presented below. The four breakout groups conducted a critical examination of the published research on BPA in relation to the four topics described above. Each of the breakout groups identified areas of knowledge and research gaps and made suggestions for future directions of research. In addition, each group identified which of the following two categories applied to specific outcomes:

- "We are confident of the following": this category applied when there were findings reported in multiple papers from multiple labs that were in agreement. There should have been no papers reporting conflicting findings, unless there were flaws in those papers, in which case the flaw(s) should have been identified.

- "We believe the following to be likely but requiring confirmation": This category applied when there were multiple consistent findings from one lab, or there may have been some conflicting reports along with reports of significant findings.

\section{Levels of confidence for published BPA findings}

The responses from the four different breakout groups were integrated together and organized based on levels of confidence. The criterion for a statement being included in a category was that there had to be consensus among all four of the breakout groups about the statement. 


\subsection{Based on existing data we are confident of the following}

\subsubsection{Issue 1: In vitro mechanistic research-laboratory animal research connection}

1. In vitro studies have provided two routes of plausibility for low dose in vivo effects of BPA. These include binding to nuclear estrogen receptors that regulate transcription as well as estrogen receptors associated with the cell membrane that promote calcium mobilization and intracellular signaling. Receptors associated with the cell membrane are more sensitive to BPA than the nuclear receptors. Actions mediated by membrane associated receptor signaling may underlie much of the low dose BPA phenomena (effects have been reported at doses as low as $1 \mathrm{pM}$ or 0.23 ppt). This increases the plausibility of effects at low doses, which are within the range of environmentally relevant doses (human and wildlife levels of exposure).

2. In vitro mechanistic information has informed us that exposing tissues to only an extremely narrow range of doses of BPA may lead to erroneous conclusions. Nonmonotonic dose-response curves are encountered frequently in basic endocrinological research, and numerous examples have been reported for BPA reviewed in Refs. [18,23,24]. Because of this animal experiments on unstudied systems must avoid narrow dose ranges, especially the use of only a few very high doses. Thus, testing one or two doses and concluding that there are no effects is inappropriate. At somewhat higher doses than are required for estrogen receptor (ER)mediated responses, BPA also interacts with androgen and thyroid hormone receptors, making predictions of effects at different doses very complex.

3. In vitro studies can dissect mechanisms of complicated effects observed in vivo. The proposed potential mechanisms acting in vitro and in vivo are the same, involving estrogen receptor mediated (nuclear- and membrane-associated) actions. However, specific effects are dose and cell/tissue specific. In addition, there are in vivo processes that are not reflective of currently known mechanisms that have been identified in vitro. This is due to previously unknown mechanisms as well as the complexity (due to interactions among cell and tissue types) of in vivo systems.

\subsubsection{Issue 2: Wildlife-laboratory animal research connection}

1. BPA is found in the environment: aquatic, terrestrial and air.

2. Studies of wildlife demonstrate estrogenic responses that are similar to responses seen in laboratory animals. Specifically, reductions in spermatogenesis are seen in wildlife at ecological concentrations of BPA, and these effects are also seen in controlled laboratory studies with BPA. In addition, vitellogenin response is a common biomarker in non-mammalian wildlife and laboratory species for BPA-induced estrogen receptor activation as well as activation by other estrogens.

3. BPA exposure induces similar effects in reproductive systems in wildlife and experimental animal model systems, but concentrations used in experiments involving wildlife species are often higher than environmental exposures. There are conditions in the environment, such as landfill leachates and effluent outflow that cause episodic exposure of field populations to elevated doses of BPA.

4. Responses in a variety of vertebrate wildlife species are qualitatively consistent with controlled laboratory studies with BPA. Thus, animals in the wild show evidence of harm, and controlled laboratory studies with model aquatic animals (i.e., medaka, zebrafish, and fathead minnows) are consistent with observations made in wildlife species. Low dose effects of BPA (low ppb range) have been observed in many of these animals. 
5. The similar effects observed in wildlife and laboratory animals exposed to BPA predict that similar effects are also occurring in humans.

\subsubsection{Issue 3: Laboratory animal research-human exposure connection}

1. Human exposure to BPA is widespread.

2. Human exposure to BPA is variable, and exposure levels cover a broad range [central tendency for unconjugated BPA: $0.3-4.4 \mathrm{ng} \mathrm{ml}^{-1}$ (ppb)] in tissues and fluids in fetuses, children and adults.

3. Because the current published literature states that there is a linear relationship between administered dose and circulating levels of BPA in animal studies, this allows circulating levels at lower administered doses to be predicted in experimental animals based on the results from studies in which higher doses were administered.

4. All of the currently published metabolic studies in rats predict circulating BPA levels after acute low dose oral exposures at blood levels less than or equal to $2 \mathrm{ng} \mathrm{ml}^{-1}$ (ppb), which is the approximate median and mean unconjugated circulating BPA level in humans. Therefore, the commonly reported circulating levels in humans exceed the circulating levels extrapolated from acute exposure studies in laboratory animals.

5. BPA levels in the fetal mouse exposed to BPA by maternal delivery of $25 \mu \mathrm{g} \mathrm{kg}^{-1}$, a dose that has produced adverse effects in multiple experiments, are well within the range of unconjugated BPA levels observed in human fetal blood.

\subsubsection{Issue 4: Life stage-relationship to exposure pharmacokinetics and health effects}

1. Sensitivity to endocrine disruptors, including BPA, varies extensively with life stage, indicating that there are specific windows of increased sensitivity at multiple life stages. Therefore, it is essential to assess the impact of life stage on the response to BPA in studies involving wildlife, laboratory animals, and humans.

2. Developmental windows of susceptibility are comparable in vertebrate wildlife species and laboratory animals.

3. BPA alters "epigenetic programming" of genes in experimental animals and wildlife that results in persistent effects that are expressed later in life [25]. These organizational effects (functional and structural) in response to exposure to low doses of BPA during organogenesis persist into adulthood, long after the period of exposure has ended. Specifically, prenatal and/or neonatal exposure to low doses of BPA results in organizational changes in the prostate, breast, testis, mammary glands, body size, brain structure and chemistry, and behavior of laboratory animals.

4. There are effects due to exposure in adulthood that occurs at low doses of BPA. Substantial neurobehavioral effects and reproductive effects in both males and females have been observed during adult exposures in laboratory animals.

5. Adult exposure studies cannot be presumed to predict the results of exposure during development.

6. Life stage impacts the pharmacokinetics of BPA. 


\subsection{We believe the following to be likely but require confirmation}

\subsubsection{Issue 1: In vitro mechanistic research-laboratory animal research connection}

1. BPA metabolism occurs in cell culture systems, and although there are differences between cell types, there is less variability than in the entire animal. Metabolism is an important issue for humans and wildlife field populations with large genetic variability. Individual differences in BPA pharmacokinetics allow for underlying variability within a population, and may allow for the identification of sensitive and insensitive subpopulations.

2. The activity of various enzymes involved in drug, chemical, and hormone metabolism, as well as protection against oxidative stress, are programmed by hormone levels during sensitive periods in development. Developmental alterations in hormonal programming (activation or inhibition) may thus affect metabolism of BPA and other hormones and chemicals. Direct interaction of BPA with enzymes in cells has only been reported at higher doses than expected for human exposures.

3. The set of genes regulated by BPA is expected to differ among doses. Therefore, different doses of BPA do not produce different effects only due to a quantitative difference in the expression of the same set of genes.

4. Differential expression of estrogen receptor subtypes ( $\alpha / \beta$; variant isoforms), and protein-protein interactions (estrogen receptor homo- and hetero-dimer formation, co-regulators, etc) modulate the cellular response to BPA. Direct actions of BPA on intracellular signal transduction modulate some cellular responses, which are similarly dependent on differential expression and protein-protein interactions.

5. Bioactive doses can be mathematically modeled, but further model refinement and experimental confirmation is required.

6. Other mechanisms (androgen receptors, thyroid hormone receptors) may be relevant for BPA action, but at higher doses than for estrogen responsive mechanisms.

\subsubsection{Issue 2: Wildlife-laboratory animal research connection}

1. The effects observed in laboratory animals could be present in wildlife, because the low doses being studied in laboratory animals are now relevant to environmental exposure levels of wildlife. The similarities in mechanisms that have been observed between different species suggest that field populations will respond to the same low levels.

2. Measurements of vitellogenin production in fish have established that there are exogenous estrogenic signals in the their environment. BPA may be contributing to this phenomenon as it enters natural water systems after leaching from landfills and due to plastic debris in water.

3. Delayed spawning is seen in male and female fish, which may relate to observed changes in estrous cyclicity in mammals in laboratory experiments.

4. In wildlife and laboratory studies, BPA induces alteration in steroid biosynthesis/ metabolism/excretion.

5. Wildlife residing in sediment is likely exposed to higher levels of BPA.

\subsubsection{Issue 3: Laboratory animal research-human exposure connection}

1. Human exposure is likely to be continuous, unlike exposure in most laboratory animal studies of BPA pharmacokinetics. 


\subsubsection{Issue 4: Life stage-relationship to exposure pharmacokinetics and health effects}

1. Clearance of BPA in the fetus is reduced compared to other life stages. Different effects and metabolic clearance mechanisms are also observed in neonatal and adult animals. Conjugation (glucuronidation) and other mechanisms of metabolic clearance of BPA thus vary throughout life.

2. Exposure to BPA during different life stages differentially influences reproductive cancer etiology and progression, and exposure during sensitive periods in organogenesis may increase susceptibility to development of cancers in some organs, such as the prostate and mammary glands.

3. Early life exposure to environmentally relevant BPA doses may result in persistent adverse effects in humans.

4. The function of the immune system can be altered following adult exposure to BPA.

5. Effects on insulin metabolism occur following adult exposure.

\subsection{Areas of uncertainty and suggestions for future research \\ 4.3.1. Issue 1: In vitro mechanistic research-laboratory animal research connection}

1. Since BPA can act as an agonist or an antagonist in different tissues and against different background physiological states, the specific co-regulators that mediated these different responses of BPA need to be elucidated based on in vitro mechanistic studies, which should be confirmed in vivo.

2. Research is needed on specific receptor sub-types (i.e., classical nuclear and nonclassical membrane-associated estrogen receptors) in relation to the potency of BPA in different tissues.

3. The identification of multiple estrogen receptor genes and variants as well as different co-regulators with different activities reveals that different levels of potency of BPA could be obtained by complex interactions between these different components that would not be predicted in homogeneous recombinant systems.

\subsubsection{Issue 2: Wildlife-laboratory animal research connection}

1. To directly relate the effects seen in wildlife with BPA exposure, biomonitoring data are needed from wildlife. In addition to BPA levels, these studies should assay total estrogenic and antiandrogenic activity from other contaminants.

2. There is a need to examine sensitive endpoints in wildlife that have been identified in laboratory animals.

3. There are substantial amounts of plastic debris within marine and fresh water ecosystems, and studies are needed to examine the impact of BPA in the environment on aquatic organisms. Doses used in laboratory experiments involving wildlife should reflect environmental exposures.

4. More studies need to be done with BPA in invertebrates, and a fundamental understanding of estrogen action in invertebrates is required.

5. Studies should determine if amplification of BPA through the food chain occurs, particularly under anaerobic or hypoxic conditions due to the lack of microbial or photodegradation. 
6. Future research emphasis should be placed on populations of aquatic animals exposed to landfill leachate and sewage effluent, as these are the primary point sources for BPA exposure.

\subsubsection{Issue 3: Laboratory animal research-human exposure connection}

1. Even though there have been attempts to estimate daily human intake of BPA, these estimates require many assumptions. The best measures we have to estimate whether humans may be affected by current exposures to BPA are levels in blood (not exposure levels), which can be related to blood levels in experimental animals after acute exposures. Known sources of human exposure to BPA do not appear sufficient to explain levels measured in human tissues and fluids.

2. While BPA is not persistent in the environment or in humans, biomonitoring surveys indicate that exposure is continuous. This is problematic because acute animal exposure studies are used to estimate daily human exposure to BPA, and at this time, we are not aware of any studies that have examined BPA pharmacokinetics in animal models following continuous low level exposures. Measurement of BPA levels in serum and other body fluids suggests that either BPA intake is much higher than accounted for, or that BPA can bioaccumulate in some conditions such as pregnancy, or both. Research using both animal models, as well as epidemiology studies, are needed to address these hypotheses, and this research needs to better mimic the apparent continuous exposure of humans to BPA.

3. More comprehensive exposure and biomonitoring studies are needed, especially in developing countries.

4. In both animal and human studies, internal exposure measures need to be related to health effects. In particular, there is a need for epidemiological studies relating health outcomes to BPA exposure, particularly during sensitive periods in development. These studies should be based on hypotheses from findings in experimental animals. This will require additional development of appropriate biomarkers in animal studies that can be used in epidemiological research.

\subsubsection{Issue 4: Life stage-relationship to exposure pharmacokinetics and health effects}

1. While there is a great need to continue studying prenatal and perinatal exposures in laboratory animal studies, many organs and endpoints continue developing at later stages (throughout puberty and adolescence). Additional studies are needed during these later periods of development.

2. Additional research is needed regarding exposure to BPA in adulthood to determine whether post-exposure effects are temporary or are permanent and associated with subsequent age-related diseases.

3. Because aging adults lose repair mechanisms, metabolic enzymes, and imprinted genes, the possibility that adult exposures (long-term, low level) can increase the risk of cancers and other conditions during aging should be addressed with additional human research and the development of appropriate animal models.

4. Epigenetics should be examined as a potential mechanism mediating developmental effects as well as the trans-generational effects of BPA and other contaminants.

Potential effects of adult exposures also need to be examined in relation to disruption of epigenetic changes that occur normally during aging. 
5. Trans- and multi-generational effects of BPA must be examined in laboratory animals and humans.

6. There is a need for studies that involve collection of human blood and urine from humans at several life stages, with specific emphasis on infants and young children and continued monitoring throughout adulthood. Additionally, there is a need to characterize the basis for the variability in BPA levels in studies examining both human urine and serum.

7. There is a need for research on the genetic basis for differences in susceptibility to BPA and other contaminants.

8. Studies are needed on comparative BPA pharmacokinetics in invertebrates and vertebrates (non-human primates included).

9. There is a need to measure total endocrine disrupter load in humans and wildlife. Therefore, biomarkers of endocrine disrupter exposure are necessary.

10. There is a need for more research directed at examining human exposure, pharmacokinetics and health effects of selected BPA precursors (i.e., BADGE, BISGMA, and BIS-DMA) and metabolites (e.g., halogenated BPAs).

11. There is a need for more studies focused on identification of other (non-estrogenreceptor mediated) mechanisms of action of BPA.

12. Effects of chemicals on the immune system are life stage dependent, and identifying the life stage dependency for BPA effects on the immune system is necessary. In addition, studies examining BPA effects on the immune system in wildlife are necessary.

\section{Conclusions}

The published scientific literature on human and animal exposure to low doses of BPA in relation to in vitro mechanistic studies reveals that human exposure to BPA is within the range that is predicted to be biologically active in over $95 \%$ of people sampled. The wide range of adverse effects of low doses of BPA in laboratory animals exposed both during development and in adulthood is a great cause for concern with regard to the potential for similar adverse effects in humans. Recent trends in human diseases relate to adverse effects observed in experimental animals exposed to low doses of BPA. Specific examples include: the increase in prostate and breast cancer, uro-genital abnormalities in male babies, a decline in semen quality in men, early onset of puberty in girls, metabolic disorders including insulin resistant (type 2) diabetes and obesity, and neurobehavioral problems such as attention deficit hyperactivity disorder (ADHD).

There is extensive evidence that outcomes may not become apparent until long after BPA exposure during development has occurred. The issue of a very long latency for effects in utero to be observed is referred to as the developmental origins of adult health and disease (DOHaD) hypothesis. These developmental effects are irreversible and can occur due to low dose exposure during brief sensitive periods in development, even though no BPA may be detected when the damage or disease is expressed. However, this does not diminish our concern for adult exposure, where many adverse outcomes are observed while exposure is occurring. Concern regarding exposure throughout life is based on evidence that there is chronic, low level exposure of virtually everyone in developed countries to BPA. These findings indicate that acute studies in animals, particularly traditional toxicological studies that only involve the use of high doses of BPA, do not reflect the situation in humans. 
The fact that very few epidemiological studies have been conducted to address the issue of the potential for BPA to impact human health is a concern, and more research is clearly needed. This also applies to wildlife, both aquatic and terrestrial. The formulation of hypotheses for the epidemiological and ecological studies can be greatly facilitated by the extensive evidence from laboratory animal studies, particularly when common mechanisms that could plausibly mediate the responses are known to be very similar in the laboratory animal models, wildlife and humans.

\section{Abbreviations}

$\begin{array}{ll}\text { ADHD } & \text { attention deficit hyperactivity disorder } \\ \text { BADGE } & \text { bisphenol A diglycidyl ether } \\ \text { BIS-DMA } & \text { bisphenol A dimethacrylate } \\ \text { BISGMA } & \text { bisphenol A glycerolate dimethacrylate } \\ \text { BPA } & \text { bisphenol A } \\ \text { ER } & \text { estrogen receptor }\end{array}$

\section{Acknowledgments}

Meeting support was provided by NIEHS and NIDCR, NIH/DHHS, the US-EPA and Commonweal. We thank Paul French for assistance with the meeting in web site and Albert Kingman for advice during preparation of the manuscript. This manuscript does not reflect US-EPA, USGS or NIH agency policy. FvS is supported by NIH grant ES11283.

\section{References}

1. Vandenberg LN, Hauser R, Marcus M, Olea N, Welshons WV. Human exposure to bisphenol A (BPA). Reprod Toxicol 2007;24:139-177. [PubMed: 17825522]

2. Wetherill YB, Akingbemi B, Kanno J, McLachlan JA, Nadal A, Sonnenschein C, et al. In vitro molecular mechanisms of bisphenol A action. Reprod Toxicol 2007;24:178-198. [PubMed: 17628395]

3. Richter CR, Birnbaum LS, Farabollini F, Newbold RR, Rubin BS, Talsness CE, et al. In vivo effects of bisphenol A in laboratory rodent studies. Reprod Toxicol 2007;24:199-224. [PubMed: 17683900]

4. Crain DA, Eriksen M, Iguchi T, Jobling S, Laufer H, LeBlanc GA, et al. An ecological assessment of bisphenol A: evidence from comparative biology. Reprod Toxicol 2007;24:225-239. [PubMed: 17604601]

5. Keri RA, Ho S-M, Hunt PA, Knudsen KE, Soto AM, Prins GS. An evaluation of evidence for the earcinogenic activity of bisphenol A. Reprod Toxicol 2007;24:240-252. [PubMed: 17706921]

6. NTP. Raleigh; NC: 2001 [Access data: June 8, 2007]. Final Report of the Endocrine Disruptors Low Dose Peer Review Panel. http://ntp.niehs.nih.gov/index.cfm?objectid=06F5CE98-E82F-8182-7FA81C02D3690D47ml

7. IRIS. Bisphenol A (CASRN 80-05-7). US-EPA Integrated Risk Information System Substance File. 1988 [Access data: June 8, 2007]. http://www.epa.gov/iris/subst/0356.htm

8. Nagel SC, vom Saal FS, Thayer KA, Dhar MG, Boechler M, Welshons WV. Relative binding affinityserum modified access (RBA-SMA) assay predicts the relative in vivo bioactivity of the xenoestrogens bisphenol A and octylphenol. Environ Health Perspect 1997;105(1):70-76. [PubMed: 9074884]

9. Colerangle JB, Roy D. Profound effects of the weak environmental estrogen-like chemical bisphenol A on the growth of the mammary gland of Noble rats. J Steroid Biochem Mol Biol 1997;60(1-2):153160. [PubMed: 9182870]

10. Steinmetz R, Brown NG, Allen DL, Bigsby RM, Ben-Jonathan N. The environmental estrogen bisphenol A stimulates prolactin release in vitro and in vivo. Endocrinol 1997;138(5):1780-1786.

11. Burridge E. Bisphenol A: product profile. Eur Chem News 2003:17. 
12. Olea N, Pulgar R, Perez P, Olea-Serrano F, Rivas A, Novillo-Fertrell A, et al. Estrogenicity of resinbased composites and sealants used in dentistry. Environ Health Perspect 1996;104(3):298-305. [PubMed: 8919768]

13. Moore, C. Synthetic polymers in the marine environment: what we know, what we need to know, what can be done?. In: Ragaini, RC., editor. Proceedings of Conference: International Seminar on Nuclear War and Planetary Emergencies, 36th Session; August 2006; Erice, Sicily. Singapore: World Scientific Publishers; in press

14. Coors A, Jones PD, Giesy JP, Ratte HT. Removal of estrogenic activity from municipal waste landfill leachate assessed with a bioassay based on reporter gene expression. Environ Sci Technol 2003;37 (15):3430-3434. [PubMed: 12966991]

15. Kawagoshi Y, Fujita Y, Kishi I, Fukunaga I. Estrogenic chemicals and estrogenic activity in leachate from municipal waste landfill determined by yeast two-hybrid assay. J Environ Monit 2003;5(2): 269-274. [PubMed: 12729267]

16. Rudel RA, Brody JG, Spengler JD, Vallarino J, Geno PW, Sun G, et al. Identification of selected hormonally active agents and animal mammary carcinogens in commercial and residential air and dust samples. J Air Waste Manage Assoc 2001;51:499-513.

17. Dodds EC, Lawson W. Synthetic oestrogenic agents without the phenanthrene nucleus. Nature 1936;137:996.

18. Welshons WV, Nagel SC, vomSaal FS. Large effects from small exposures. III. Endocrine mechanisms mediating effects of bisphenol A at levels of human exposure. Endocrinology 2006;147 Suppl. 6:S56-S69. [PubMed: 16690810]

19. Quesada I, Fuentes E, Viso-Leon MC, Soria B, Ripoll C, Nadal A. Low doses of the endocrine disruptor bisphenol-A and the native hormone 17beta-estradiol rapidly activate transcription factor CREB. FASEB J 2002;16(12):1671-1673. [PubMed: 12207000]

20. Walsh DE, Dockery P, Doolan CM. Estrogen receptor independent rapid non-genomic effects of environmental estrogens on $\left[\mathrm{CA}^{2+}\right]_{\mathrm{i}}$ in human breast cancer cells. Mol Cell Endocrinol 2005;230(12):23-30. [PubMed: 15664448]

21. Wozniak AL, Bulayeva NN, Watson CS. Xenoestrogens at picomolar to nanomolar concentrations trigger membrane estrogen receptor-a mediated $\mathrm{Ca}++$ fluxes and prolactin release in GH3/B6 pituitary tumor cells. Environ Health Perspect 2005;113:431-439. [PubMed: 15811834]

22. Zsarnovszky A, Le HH, Wang HS, Belcher SM. Ontogeny of rapid estrogen-mediated extracellular signal-regulated kinase signaling in the rat cerebellar cortex: potent nongenomic agonist and endocrine disrupting activity of the xenoestrogen bisphenol A. Endocrinol 2005;146(12):5388-5396.

23. vom Saal FS, Welshons WV. Large effects from small exposures. II. The importance of positive controls in low-dose research on bisphenol A. Environ Res 2006;100:50-76. [PubMed: 16256977]

24. Welshons WV, Thayer KA, Judy BM, Taylor JA, Curran EM, vom Saal FS. Large effects from small exposures. I. Mechanisms for endocrine-disrupting chemicals with estrogenic activity. Environ Health Perspect 2003;111(8):994-1006. [PubMed: 12826473]

25. Ho SM, Tang WY, Belmonte de Frausto J, Prins GS. Developmental exposure to estradiol and bisphenol A increases susceptibility to prostate carcinogenesis and epigenetically regulates phosphodiesterase type 4 variant 4. Cancer Res 2006;66(11):5624-5632. [PubMed: 16740699] 